\title{
Article
}

\section{Pseudomonas aeruginosa antibiotic efflux pump variants exhibit increased virulence}

\author{
Mylene Vaillancourt ${ }^{1}$, Sam P. Limsuwannarot ${ }^{2}$, Catherine Bresee ${ }^{3}$, Rahgavi Poopalarajah ${ }^{4}$ and Peter Jorth ${ }^{5 *}$ \\ 1 Department of Pathology and Laboratory Medicine, Cedars-Sinai Medical Center, Los Angeles, CA, USA; \\ Mylene.Vaillancourt@cshs.org \\ 2 Department of Pathology and Laboratory Medicine, Cedars-Sinai Medical Center, Los Angeles, CA, USA; \\ Podsawee.Limsuwannarot@cshs.org \\ 3 Biostatistics Core, Cedars-Sinai Medical Center, Los Angeles, CA, USA; Catherine.Bresee@cshs.org \\ 4 Department of Biological Sciences, University of Calgary, Calgary, AB, Canada; Rahgavi.Poopalara- \\ jah@ucalgary.ca \\ 5 Department of Pathology and Laboratory Medicine, Dept. of Medicine, Dept. of Biomedical Sciences, Ce- \\ dars-Sinai Medical Center, Los Angeles, CA, USA; Peter.Jorth@cshs.org \\ * Correspondence: Peter.Jorth@cshs.org; Tel.: +1-310-967-2712
}

\begin{abstract}
Antibiotic resistant Pseudomonas aeruginosa infections are the primary cause of mortality in people with cystic fibrosis (CF). Yet it has only recently become appreciated that resistance mutations can also increase $P$. aeruginosa virulence, even in the absence of antibiotics. Moreover, the mechanisms by which resistance mutations increase virulence are poorly understood. In this study we tested the hypothesis that mutations affecting efflux pumps can directly increase $P$. aeruginosa virulence. Using genetics, physiological assays, and model infections, we show that efflux pump mutations can increase virulence. Mutations of the mexEF efflux pump system increased swarming, rhamnolipid production, and lethality in a mouse infection model, while mutations in mexR that increased expression of the mex $A B$-opr $M$ efflux system increased virulence during an acute murine lung infection without affecting swarming or rhamnolipid gene expression. Finally, we show that an efflux pump inhibitor, which represents a proposed novel treatment approach for $P$. aeruginosa, increased rhamnolipid gene expression in a dose-dependent manner. This finding is important because rhamnolipids are key virulence factors involved in dissemination through epithelial barriers and cause neutrophil necrosis. Together, these data show how current and proposed future antiPseudomonal treatments may unintentionally make infections worse by increasing virulence. Therefore, treatments that target efflux should be pursued with caution.
\end{abstract}

Keywords: Pseudomonas, Efflux Pumps, Virulence, Evolution, Antibiotic Resistance, Cystic Fibrosis

\section{Introduction}

Pseudomonas aeruginosa is a ubiquitous Gram-negative bacterium that colonizes a wide range of environments [1]. This environmental flexibility is due to its high adaptability to changing conditions and is driven by substantial metabolic versatility, expression of a large array of virulence factors, and extensive adaptative transport and efflux systems [2]. $P$. aeruginosa is infamous for causing serious nosocomial infections such as burn wound infections and ventilator-associated pneumonia [3] and it is the predominant pathogen causing chronic infections in cystic fibrosis (CF) [4].

During chronic infections, $P$. aeruginosa adapts to the CF lung environment by decreasing production of virulence factors and evolves to become antibiotic resistant [5-7]. Even more complicated, populations of $P$. aeruginosa within patients evolve high degrees of diversity and within-patient variability, where contemporary sibling cells that have descended from a common ancestor can differ in antibiotic susceptibilities and virulence 
factor production [8-11]. P. aeruginosa virulence mechanisms include attachment to cells (e.g., pili), induction of host damage (e.g., DNase, proteases), competition with the host for nutrients, and secretion of surfactant molecules such as rhamnolipids which cause neutrophil necrosis and permit dissemination [12-14]. Virulence factors usually trigger an exacerbated inflammatory response by the innate immune system, with macrophages and neutrophils as the first line of defense [15]. Thus, it has been proposed that decreasing virulence factor production attenuates host inflammatory response and favors bacterial colonization of the lung during CF chronic infections.

In addition to the host pressures that affect virulence, bacteria colonizing CF lungs also experience intense antibiotic pressure due to mono and combination therapies including aminoglycosides, quinolones, and $\beta$-lactams [16]. This environment strongly favors selection of antibiotic resistant strains. Some of the best characterized antibiotic resistance features are efflux pump systems that expel antibiotics out of the cell. For example, we previously showed that nalD and mexR genes, both encoding for transcriptional repressors of the MexAB-OprM efflux pump [17-19], were recurrently mutated under aztreonam selection [20]. P. aeruginosa with mutations in nalD and mexR overexpress the MexAB-OprM efflux pump, rendering them multidrug resistant [17, 19, 21]. Moreover, mutations in these two genes have been found in anywhere from $7-47 \%$ of clinical isolates in various studies [20-24]. Importantly, strains with mutations in nalD and mexR evolved under aztreonam selection showed an increased virulence in vivo, challenging the longstanding theory that antibiotic resistance mutations inherently pay fitness costs that attenuate virulence (Jorth et al., 2017). However, whether these mutations increase virulence in an efflux-dependent manner remains unknown.

In this study, we sought to investigate mechanisms involved in the hypervirulence of a mutant P. aeruginosa strain, PAO1-AzEvB8, that was experimentally evolved in a previous study during cyclic aztreonam exposure [20]. Because this strain was previously shown to have a mutation in mexR, we hypothesized that its hypervirulence could be attributed to increased expression of the MexAB-OprM efflux pump. We explore this hypothesis using a combination of genome sequencing, genetics, transcriptional reporter assays, and infection models.

\section{Results}

\subsection{Deletion of mexAB restores aztreonam susceptibility of evolved P. aeruginosa}

Previously, we used experimental evolution with cyclical exposure to aztreonam to evolve the aztreonam resistant strain PAO1-AzEvB8 [20]. Strain PAO1-AzEvB8 was found to have a nonsense mutation in mexR (E118*) and complementation with a wild-type (WT) $\operatorname{mexR}$ gene restored aztreonam susceptibility [20]. Because mexR mutations have been shown to increase expression of the mexAB-oprM efflux pump operon $[19,21]$, which encodes the pump that effluxes aztreonam, we hypothesized that deletion of mex $A B$ in PAO1-AzEvB8 would restore aztreonam susceptibility. We generated clean mex $A B$ deletions in both WT PAO1 and in PAO1-AzEvB8, and the PAO1-AzEvB8 $\triangle$ mexAB strain was equally susceptible to aztreonam by a gradient diffusion assay as the PAO1 $\triangle$ mex $A B$ strain (Figure 1A); the mode minimum inhibitory concentrations (MIC) for $\triangle m e x A B$ strains was $0.19 \mu \mathrm{g} / \mathrm{ml}$, compared to $16 \mu \mathrm{g} / \mathrm{ml}$ for PAO1-AzEvB8 and $1.0 \mu \mathrm{g} / \mathrm{ml}$ for WT PAO1. This showed that mexAB was required for aztreonam resistance of the of the PAO1-AzEvB8 mutant. 

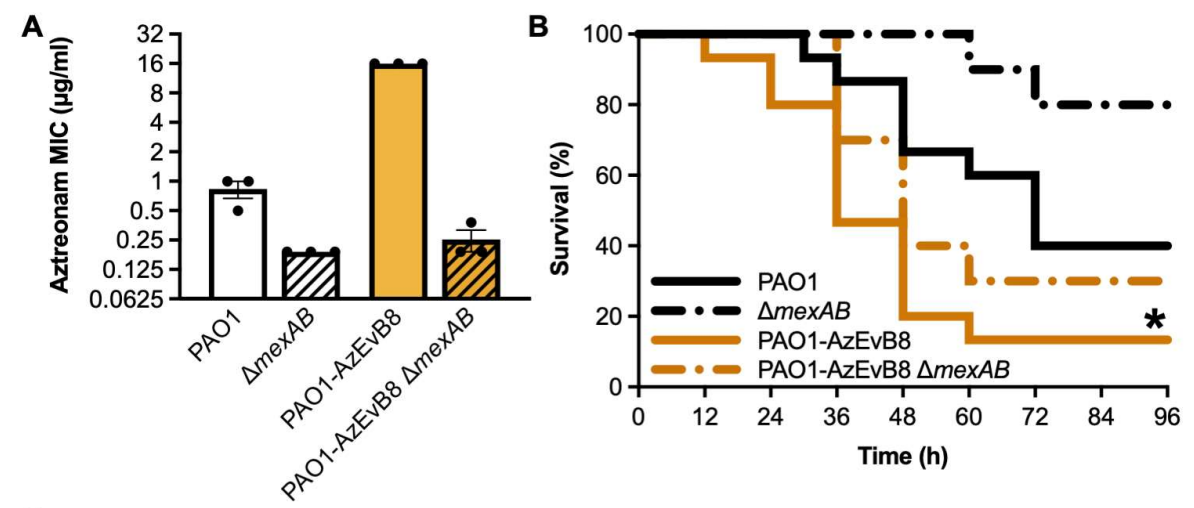

C

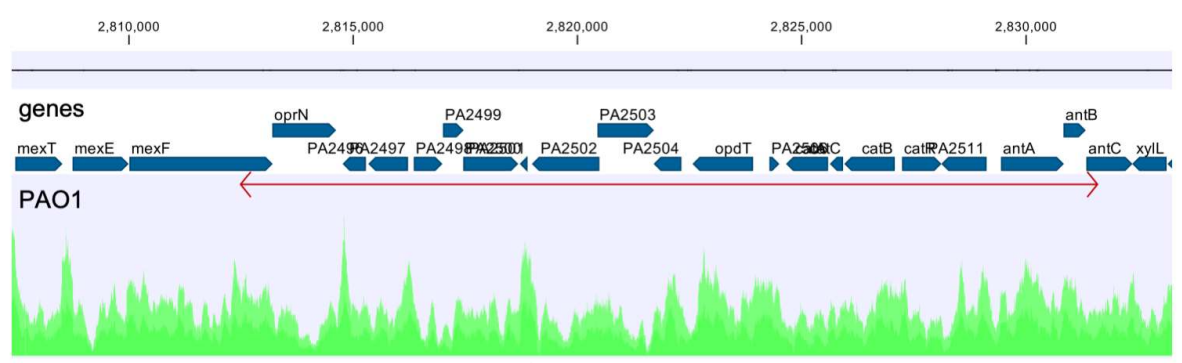

PAO1 $\triangle \operatorname{mexAB}$

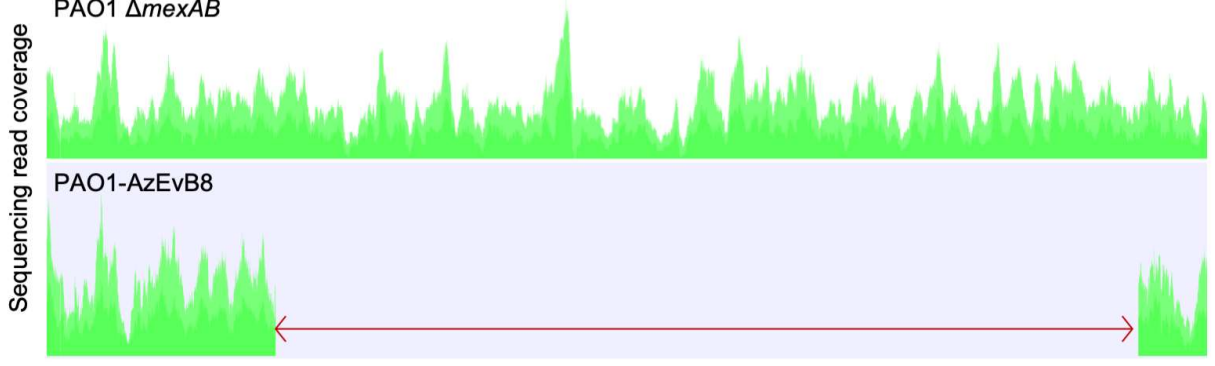

PAO1-AzEvB8 $\triangle \operatorname{mexAB}$

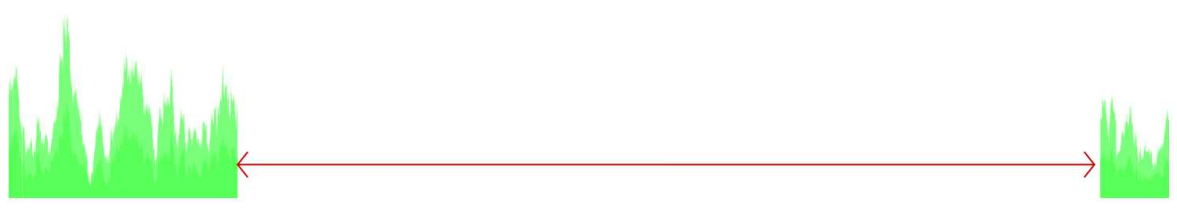

Figure 1. MexAB-OprM is not involved in the PAO1-AzEvB8 mutant in vivo virulence. (a) Deletion of the mex $A B$ genes abrogates aztreonam resistance in strain PAO1-AzEvB8. Aztreonam MICs were determined by gradient diffusion assays ( $\mathrm{n}=3$ replicates/group; MIC: minimum inhibitory concentration). (b) mexAB deletion in PAO1-AzEvB8 mutant does not significantly improve mouse survival following acute lung infection. Log-rank test was used to compare survival curves. ${ }^{*} p<0.05$ compared to WT PAO1; $\mathrm{n}=10-15$ mice/group. (c) Genome diagram showing coverage of sequencing reads aligning to the region spanning mexEF through $x y l Z$. The 19,233 bp deleted region (indicated by the red arrow) in strains PAO1-AzEvB8 and PAO1-AzEvB8 $\triangle$ mexAB begins at the $3^{\prime}$ end of mexF and continues through the $5^{\prime}$ region of antC. Genome coverage plots generated from sequencing read alignments to the PAO1 reference genome are indicated in green.

\subsection{Deletion of mexAB does not affect virulence of evolved P. aeruginosa}

In addition to exhibiting increased aztreonam resistance relative to WT PAO1, strain PAO1-AzEvB8 also displayed increased killing of mice in an acute murine infection model. We hypothesized that, similar to the aztreonam resistance, the increased virulence of PAO1-AzEvB8 would be dependent upon mexAB which was overexpressed in this strain. Surprisingly, the PAO1-AzEvB8 $\triangle \operatorname{mex} A B$ strain was equally virulent to strain PAO1-AzEvB8 (Figure 1B), indicating that the increased virulence was not dependent on $m e x A B$ alone. This was even more unexpected since PAO1 $\triangle m e x A B$ was less virulent than 
WT PAO1 (Figure 1B). This suggested that another gene or mutation was involved in the enhanced virulence of PAO1-AzEvB8.

\subsection{Genome sequencing reveals a previously undetected $19 \mathrm{~kb}$ deletion in PAO1-AzEvB8}

To determine whether another mutation may be present in strain PAO1-AzEvB8, we performed whole genome sequencing on strains PAO1 (WT parent strain), PAO1 $\triangle$ mexAB, PAO1-AzEvB8, and PAO1-AzEvB8 $\triangle$ mexAB. Using this approach, we found that relative to WT PAO1, a large $\sim 19 \mathrm{~kb}$ deletion affecting 21 genes spanning mex $F$ through ant $C$ was present in the PAO1-AzEvB8 and PAO1-AzEvB8 $\triangle$ mexAB strains (Figure 1C). This deletion was not detected by the genome sequencing in our original study despite $>10$-fold sequencing coverage of the genome [20]. The genes affected by this mutation included $m e x F$ and $o p r N$ which form part of the mexEF-oprN efflux pump operon. Previously, these genes have been linked to the swarming phenotype in $P$. aeruginosa, with studies indicating that strains overexpressing mexE have decreased swarming $[25,26]$. This led us to predict that the mexF mutation could lead to increased swarming and rhamnolipid production in the PAO1-AzEvB8 strain.

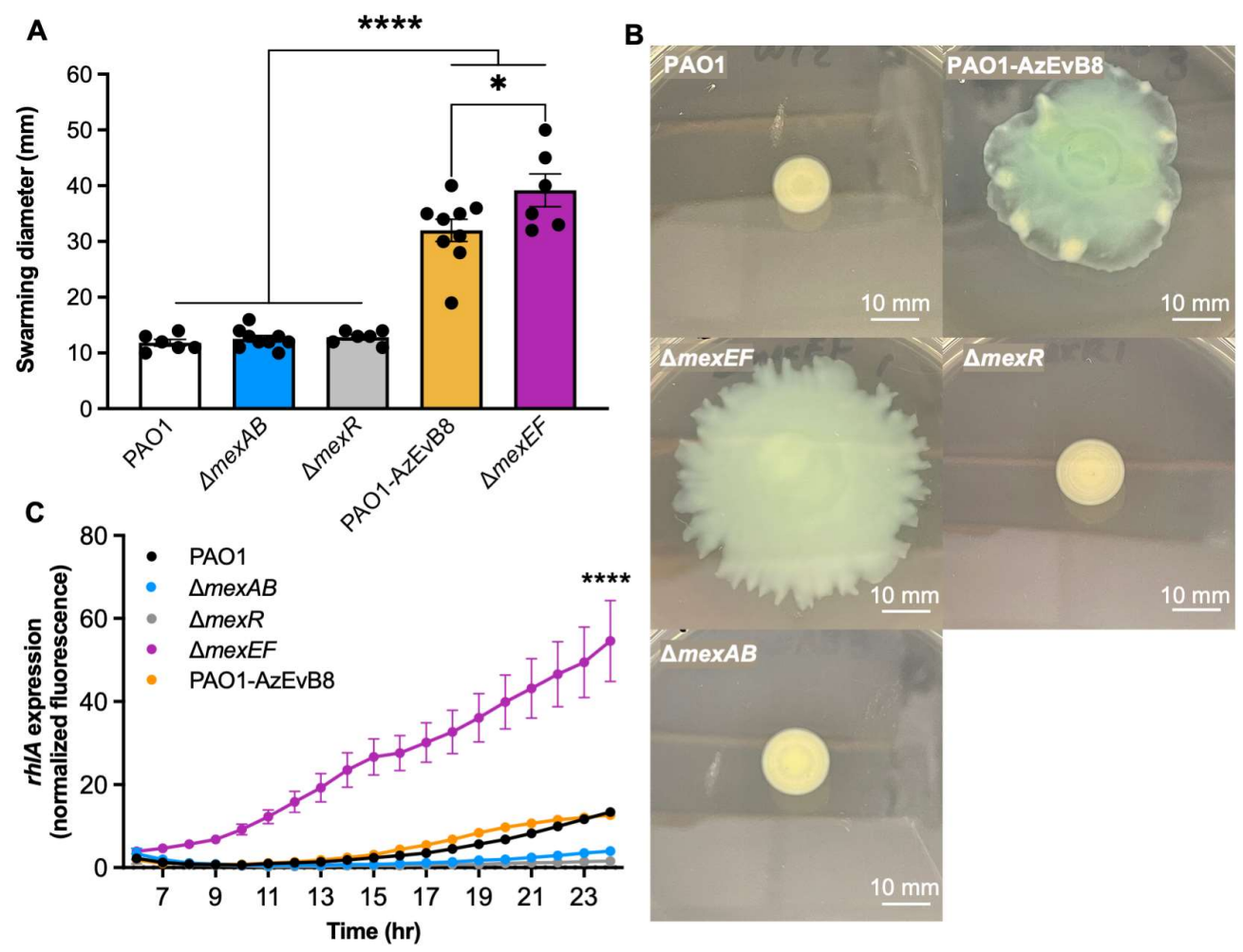

Figure 2. MexEF-OprN deletion increases swarming motility and biosurfactant production. (a) Swarming is increased in the PAO1-AzEvB8 and $\triangle m e x E F$ strains. Measurement of the swarming motility in different mexAB-oprM and mexEF-oprN mutants. One-way ANOVA was used followed by a Tukey's multiple comparisons test. ${ }^{* * * *} p<0.0001 ; n=6-7$ replicates/group. (b) Representative images of swarming motility from (a). Scale bars indicate $10 \mathrm{~mm}$. (c) Rhamnolipid gene expression is increased in the $\triangle m e x E F$ strain. Rhamnolipid production measured by quantification of rhlA gene expression using a $r h l A-g f p$ promoter reporter fusion to measure GFP fluorescence over time. Gene expression was calculated as area under the curve for each strain $\left({ }^{* * *} p<0.0001\right.$ compared to all other groups, one-way ANOVA followed by a Tukey's multiple comparisons test, $n=6-7$ replicates/group). 
Based on the deletion of a large part of the mexEF-oprN operon in strain PAO1AzEvB8, we hypothesized that this strain would exhibit increased swarming, as would mutants lacking mexEF. To test this, we generated a strain with the mexEF genes deleted in a WT PAO1 background. Gradient diffusion susceptibility testing showed that the PAO1 $\triangle$ mexEF strain was more susceptible to both ciprofloxacin and chloramphenicol than WT PAO1 (mode ciprofloxacin MICs: PAO1 $\Delta$ mexEF $0.125 \mu \mathrm{g} / \mathrm{ml}$ vs WT PAO1 0.5 $\mu \mathrm{g} / \mathrm{ml}$; mode chloramphenicol MICs: PAO1 $\Delta$ mexEF $48 \mu \mathrm{g} / \mathrm{ml}$ vs WT PAO1 >256 $\mu \mathrm{g} / \mathrm{ml}$; $\mathrm{n}=3$ ). Swarming assays were performed using the WT PAO1, PAO1-AzEvB8, PAO1 $\triangle m e x E F$, and PAO1 $\triangle m e x R$ strains. As predicted, the PAO1-AzEvB8 strain displayed more swarming than WT, as did PAO1 $\triangle$ mexEF (Figure 2AB). In contrast, deletion of mexR in a WT PAO1 background did not affect swarming (Figure 2AB). Next, we tested whether mexEF mutations led to increased rhamnolipid production, because rhamnolipids are involved in swarming and cause necrosis of neutrophils [13]. A rhlA-gfp transcriptional reporter was transformed into strains PAO1, PAO1 $\triangle$ mexEF, PAO1 $\triangle$ mexAB, PAO1 $\triangle m e x R$, and PAO1-AzEvB8 and rhlA expression was monitored during the course of growth. Consistent with the swarming phenotypes, PAO1 $\triangle$ mexEF had the highest $r h l A$ expression (Figure 2C). However, unexpectedly, PAO1-AzEvB8 had less rhlA expression than PAO1 $\triangle m e x E F$, similar rhlA expression to WT PAO1, and slightly more rhlA expression than PAO1 $\triangle$ mex $A B$ and PAO1 $\triangle$ mexR strains (Figure 2C). Together these data showed that the PAO1-AzEvB8 strain had a hyper swarming phenotype, a mexEF deletion mutation was sufficient to increase swarming, and a mexR deletion mutation alone does not affect swarming.

\subsection{Deletion of either mexR or mexEF increases $P$. aeruginosa virulence}

To better understand the increased virulence of the PAO1-AzEvB8 strain, we analyzed the virulence of PAO1 $\triangle m e x R$ and PAO1 $\triangle m e x E F$ strains to determine if either the mexR or mexF mutations detected in the PAO1-AzEvB8 strain could have contributed to the increased virulence. Using an acute murine lung infection model, we found that both PAO1 $\triangle m e x R$ and PAO1 $\triangle m e x E F$ killed the infected mice significantly faster than the WT PAO1 strain (Figure 3). These findings suggest that either of the two mutations present in the PAO1-AzEvB8 were sufficient to increase virulence relative to the WT parent strain.

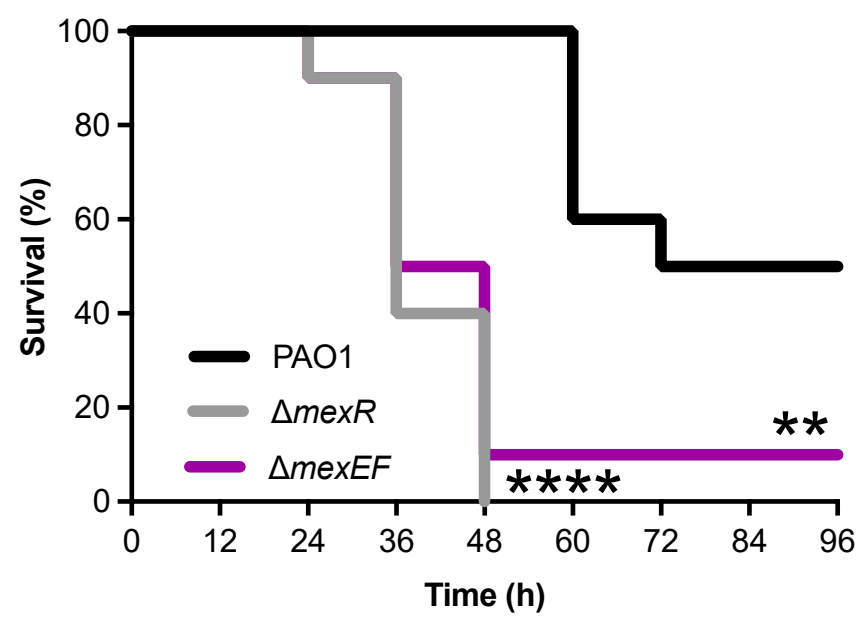

Figure 3. MexAB-OprM overexpression and MexEF-OprN deletion exhibit increased in vivo virulence. Both $\triangle m e x R$ and $\triangle m e x E F$ mutations in WT PAO1 significantly reduce mouse survival during an acute lung infection compared to WT PAO1 (WT). Survival times were tested across strata with a Bonferroni-adjusted log-rank test: ${ }^{* *} p<0.005$ and ${ }^{* * *} p<0.0001$ compared to WT PAO1, $\mathrm{n}=10$ mice/group.

\subsection{Efflux pump inhibition increases rhamnolipid virulence factor expression}


The swarming assays and mouse virulence experiment suggested that loss of the mexEF efflux pump was sufficient to increase swarming, rhamnolipid expression, and virulence in a mouse lung infection model. Therefore, we hypothesized that inhibition of efflux pump activity would lead to increased rhamnolipid gene expression. To test this, we exposed the PAO1 attB::rhlA-gfp reporter strain to increasing concentrations of the PA $\beta N$ efflux pump inhibitor [27]. As hypothesized, increasing concentrations of PA $\beta N$ from $0-25 \mu \mathrm{g} / \mathrm{ml}$ led to increased $r h l A$ rhamnolipid gene expression in a dose-dependent manner (Figure 4). This showed that a broad-spectrum efflux pump inhibitor could increase expression of an important virulence factor.

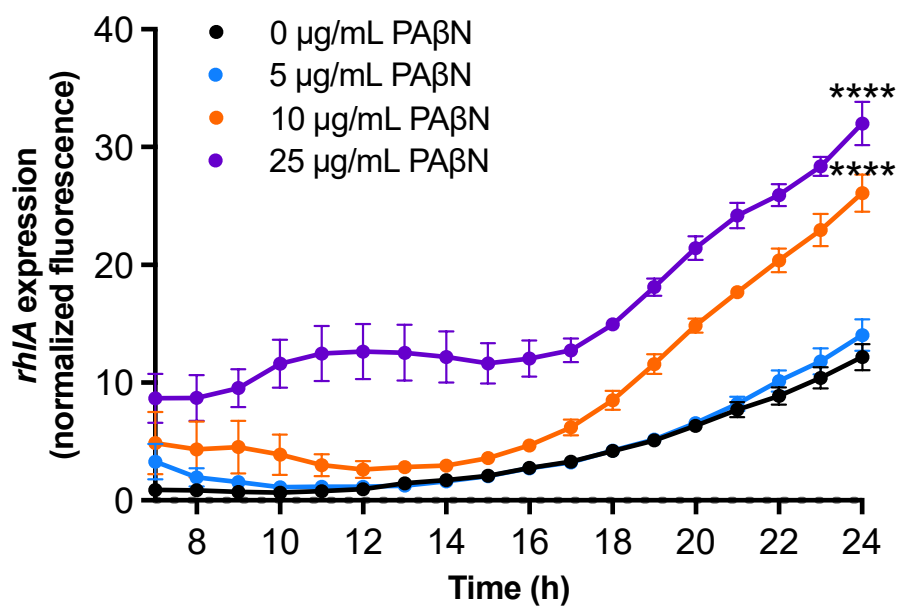

Figure 4. Rhamnolipid gene expression is induced in WT PAO1 by the Pa $\mathrm{NN}$ efflux pump inhibitor. rhlA expression in WT PAO1 attB::rhlA-gfp treated with $0-25 \mu \mathrm{g} / \mathrm{mL} \mathrm{Pa} \beta \mathrm{N}$ as measured by GFP fluorescence from the $a t t B:: r h l A-g f p$ promoter reporter fusion over time. Gene expression was calculated as area under the curve for each concentration ${ }^{* * * *} p<0.0001$ compared to all other groups, one-way ANOVA followed by a Tukey's multiple comparisons test., $\mathrm{n}=6$ replicates/group).

\section{Discussion}

Here we showed that two different mutations affecting efflux pumps that evolved in response to aztreonam selective pressure can increase $P$. aeruginosa virulence in the absence of antibiotic treatment. This finding is interesting because one mutation, mex $R$, leads to overexpression of the MexAB-OprM efflux pump, while the other mutation conferred the loss-of-function of a second efflux pump, mexEF-oprN. We also showed that deletion of mexEF caused increased swarming, likely due to increased $r h l A$ gene expression, which was demonstrated using a rhamnolipid gene expression reporter. Finally, we showed that efflux pump inhibition can have the unexpected effect of increasing rhamnolipid gene expression. This observation is important because rhamnolipids can cause necrosis of neutrophils and are also involved in the penetration of $P$. aeruginosa through epithelial barriers $[13,14]$. Altogether these data highlight how antibiotic selection can unexpectedly increase virulence through multiple pathways.

One curious result was that the PAO1-AzEvB8 strain did not overexpress rhlA relative to WT PAO1, despite exhibiting increased swarming. Because this strain had a large deletion mutation that included the $3^{\prime}$ end of mexF, we expected it to exhibit elevated rhlA expression similar to the PAO1 $\triangle m e x E F$ strain. One possible explanation is that with an intact mexE gene, the increase in $r h l A$ relative to WT may be less pronounced than when both mexE and mexF are deleted. This is somewhat apparent in the quantification of the swarming assays where the swarming zone of PAO1 $\triangle m e x E F$ was slightly larger than PAO1-AzEvB8. Another possible explanation is that the swarming assays were performed using semi-solid $0.5 \%$ agar plates, whereas the $r h l A$ expression assays were done in broth 
cultures. In future work we plan to explore whether differences in $r h l A$ expression are detectable during infection, which is where it is likely most relevant.

Overall, these results are consistent with previous studies which found that $P$. aeruginosa $n f x C$ mutants, which overexpress mexEF-oprN, do not swarm and exhibit decreased rhlA expression relative to WT [26]. Likewise, these data also align with a study by Cosson et al. which found that $P$. aeruginosa $n f x C$ mutants were avirulent in a rat acute pneumonia infection model, and virulence could be partially restored in the $n f x C$ mutant by deleting mexE [28]. An important distinction is that, to our knowledge, it had not previously been shown that deletion of mexEF in a WT genetic background can increase virulence.

While our data agree with the studies discussed above, the finding that the $\triangle m e x E F$ strain was more virulent than WT in the acute murine lung infection does appear to conflict other previous research related to efflux pump inhibition. In a study from Hiraka et al., PA $\beta \mathrm{N}$ was shown to reduce intracellular invasion of Madin-Darby canine kidney epithelial cell monolayers by $P$. aeruginosa [29], which appears inconsistent with our findings. One simple explanation for this difference is that we were not measuring intracellular invasion in our model. Instead, we measured virulence by survival following acute pneumonia, which we argue is more similar to human lung infections because neutrophils and epithelial barriers are present that can be harmed by the excess rhamnolipid produced in mexEF mutants and bacteria treated with PA $\beta N$. In another study, PA $\beta N$ reduced swarming and killing of Galleria mellonella larvae [30]. Again, slight differences in the infection model and the swarming assay could help explain differences from the present study. For example, the media used for the swarming assay are different from the present study [30]. The G. mellonella infection model is also quite distinct from the mouse infection model used here, as it seems to be primarily affected by type III secretion [31] and lipopolysaccharide [32], and the role of rhamnolipids in the G. mellonella model is less clear. Therefore, we believe that differences in experimental design can account for the differences in interpretation of the effects of the PA $\beta N$ efflux pump inhibitor. Future experiments will be required to determine whether PA $\beta N$ and similar efflux pump inhibitors enhance or decrease virulence in mammalian infections.

While mexR mutations have been identified in countless $P$. aeruginosa clinical isolates in previous studies [24, 33], mutations directly affecting mexEF have not been as commonly described. Previous work has shown that mutations in mexT, the transcriptional activator of mexEF, are very common and these mutants were dubbed $n f x C$ type mutants for their resistance to norfloxacin $[34,35]$. Additionally, several studies have also examined mexE gene expression in large sets of clinical isolates, and in some strains mexE expression was reported to be less than WT or completely undetected [22, 36, 37]. This suggests that these strains have either lost function of mexT or may potentially have deletions in mexE. This raises the possibility that these clinical isolates would also exhibit increased swarming, increased rhamnolipid production, and be more virulent than other clinical isolates, though this has not yet been investigated. We plan to test these phenotypes in clinical isolates with reduced mexE expression in the future.

These results have several implications that are particularly relevant to CF treatment, including those for currently used anti-Pseudomonal therapies, as well as proposed new therapies.

First, these data show how aztreonam, a common antibiotic used to treat $P$. aeruginosa infections in people with CF, can select for not one, but two different mutations that increase $P$. aeruginosa virulence. This may help explain why a recent clinical observational study found that people with CF that were infected with aztreonam resistant $P$. aeruginosa were more likely to experience pulmonary exacerbations and be hospitalized than people infected with aztreonam susceptible $P$. aeruginosa [38]. If the isolates in those patients evolved mutations in either mexR or mexEF-oprN, then this could lead to increased virulence and lung damage in these individuals. Future work investigating the prevalence and association of these mutations with clinical outcomes will help test this theory.

Second, these data show that efflux pump inhibitors might have unexpected negative consequences on infections in people. Efflux pump inhibitors like PA $\beta N$ can inhibit efflux 
and potentiate other antibiotics that would normally be expelled through those pumps [27]. However, the experiments presented here show that inhibiting or deleting one efflux pump, MexEF-OprN, can have the unintended consequence of increasing rhamnolipid gene expression. Thus, inhibitors that affect MexEF-OprN may not be good candidates for use clinically. Instead, it would be more beneficial to identify more specific efflux pump inhibitors. For example, the MexAB-OprM-specific efflux pump inhibitor D13-9001 [39] could be used to block MexAB-OprM without inhibiting MexEF-OprN, and this could make $P$. aeruginosa more susceptible to other antibiotics and reduce virulence, since mex $A B$ deletion mutants are less virulent than WT $P$. aeruginosa, as shown here and in other research [40]. Our data highlight the need to explore these types of precise approaches in future research using animal infection models.

It is also important to acknowledge the limitations of this work. First, strain PAO1AzEvB8 that was the focus of the present study was not an actual clinical isolate and it is unclear how frequently mutants arise that have both mex $R$ and mexEF mutations. As mentioned above, literature suggests that $\operatorname{mex} R$ mutations are very common in $\mathrm{CF}$, and that clinical isolates can display varying degrees of mexE gene expression [20, 22, 24, 33, 36, 37], so we feel that this study still has relevance to real-world P. aeruginosa infections.

The second primary limitation to this work is that the mouse model analyzed does not perfectly model the chronic infections that affect people with CF. One weakness is that this model does not capture the types of nutrient and biochemical properties present in $C F$ that can affect $P$. aeruginosa antibiotic tolerance and virulence factor expression [41-44]. However, one strength to this model is that it does recapitulate the airway epithelial cell barrier and neutrophils present in CF infections that are susceptible to rhamnolipid-mediated toxicity $[13,14]$. To explore effects of these mutations on other aspects of CF lung disease, future work could utilize chronic infection models in rodents or the more recently developed CF ferret and pig models [14, 45-48].

One final limitation to this work is that the clean deletion mutation in the PAO1 $\triangle m e x E F$ strain was not generated in-frame with the mex $E$ and mex $F$ coding sequences, whereas the mexR and mex $A B$ deletion constructs were generated in-frame with the original genes. Therefore, it is possible that a small peptide may be transcribed in the $\triangle m e x E F$ strain that is out-of-frame $(+1)$ relative to the mexEF-oprN coding sequences. This could have two consequences independent of the mexEF deletion. First, the $\triangle m e x E F$ strain phenotypes could be caused by this novel peptide and not the mexEF deletion which was confirmed by whole genome sequencing. We think this is unlikely because the other phenotypes of this strain, including increased antibiotic susceptibilities to ciprofloxacin and chloramphenicol are consistent with previous studies of mexE mutants [26, 35]. The other possibility is that these phenotypes could be caused by effects of the mexEF deletion that prevent normal expression of oprN. Even if this were true, the phenotypes would still be consistent with the overall loss-of-function of the mexEF-oprN efflux pump, which is consistent with our conclusions. Long-term we plan to perform complementation studies to tease out whether the swarming, rhamnolipid, and virulence phenotypes are due to lossof-function of all three genes in the mexEF-oprN operon, or if phenotypes can be caused by mutation by each of the individual genes.

Altogether this work sheds important light on how antibiotic resistance mutations can affect efflux pumps and subsequent $P$. aeruginosa virulence. Long-term, we believe that new strategies for treating efflux pump overexpressing or deletion mutants should be pursued. Additionally, we plan to explore how mexR mutations lead to increased virulence, since this mechanism still remains mysterious.

\section{Materials and Methods}

\subsection{Bacterial Strains and Growth Conditions}

Bacterial strains and plasmids are listed in Table S1. Mutant strains were derived from WT PAO1, which was obtained from Colin Manoil's laboratory at the University of 
Washington [49]. Strains were routinely grown at $37^{\circ} \mathrm{C}$ on LB agar and in LB broth unless otherwise specified.

\subsection{Deletion plasmid construction}

PAO1 strains carrying full deletions of mex $A B$, mexEF, or mexR genes listed in Table $S 1$ were generated with a suicide plasmid as described previously [50]. For the $\triangle m e x A B$ and $\triangle$ mexR strains, two PCR fragments were generated from chromosomal DNA for each construct using the following primer pairs up- and down-stream of each target for mutation; mex $A B$ genes: mexAB-KO-UP-F and mexAB-KO-UP-R for the upstream mex $A B$ fragment and mexAB-KO-DN-F and mexAB-KO-DN-R for the downstream mex $A B$ fragment; and mexR: mexR-KO-UP-F and mexR-KO-UP-R for the upstream mexR fragment and mexR-KO-DN-F and mexR-KO-DN-R for the downstream mexR fragment. The suicide plasmid pEX18Gm [51] was prepared for assembly by restriction digest or PCR amplification with primers pEX18Gm-F and pEX18Gm-R (Table S1). The two fragments for each construct were then assembled into the prepared vector pEX18Gm [51] using NEBuilder HiFi DNA Assembly Cloning Kit (cat\# E5520) for the $\triangle$ mex AB mutant and the NEB Gibson Assembly Master Mix (cat\# E2611L) for the $\Delta m e x R$ mutant. Suicide plasmids were transformed into E. coli DH5 $\alpha$ [52] competent cells (NEB cat\# C2987H) according to manufacturer's protocol, selected on LB agar with $10 \mu \mathrm{g} / \mathrm{mL}$ gentamicin (Gm). Plasmids were isolated using the NEB Monarch Plasmid Purification MiniPrep Kit (cat\# T1010L) and verified by Sanger sequencing. For the $\triangle m e x E F$ mutant construct, two sets of primers (oRP_21, oRP_22 \& oRP_23, oRP_24) were designed to amplify 527 and 495 bp regions upstream and downstream, respectively, of the mexEF genes. These PCR fragments were assembled by splicing by overlap extension PCR and the deletion allele, containing attachment sites (attB1- and attB2-) for Gateway recombination, was integrated into the pDONRPEX18Gm vector via the BP Clonase reaction, as previously described (Hmelo et al., 2015). The reaction mixture was transformed into $E$. coli DH5 $\alpha$ by electroporation, and clones harboring the plasmid with the $\triangle m e x E F$ allele were selected on LB agar with $10 \mu \mathrm{g} / \mathrm{ml} \mathrm{Gm}$ and identified by colony PCR using M13 universal primers. This yielded plasmid pRP12 (pDONRPEX18Gm:: $\triangle m e x E F$ ), which was sequence verified using M13 primers.

\subsection{Transformation of $P$. aeruginosa deletion mutants}

PAO1 deletion mutants were generated from WT P. aeruginosa PAO1 through twostep allelic exchange through either electroporation or mating, as described previously [50]. For electroporation of pEX18Gm:: $\operatorname{mex} A B, 1.5 \mathrm{~mL}$ of PAO1 or PAO1-AzEvB8 grown overnight in LB were centrifuged at $14,000 \mathrm{~g}$ for $3 \mathrm{~m}$ and washed twice with $300 \mathrm{mM}$ sterile sucrose. Cells were then centrifuged at $14,000 \mathrm{~g}$ for $3 \mathrm{~m}$ and resuspended in $100 \mu \mathrm{L}$ of 300 $\mathrm{mM}$ sterile sucrose. One microgram of pEX18Gm:: $\triangle$ mex $A B$ was then added to each cell suspension, and the bacterial suspensions were transformed by electroporation at $2.5 \mathrm{kv}$ and incubated on LB with $30 \mu \mathrm{g} / \mathrm{mL} \mathrm{Gm}$ at $37^{\circ} \mathrm{C}$ overnight. For counter selection, isolated clones were streaked on low-salt LB containing 15\% sucrose as published [50] and incubated at room temperature for $48 \mathrm{~h}$. Gene deletions were confirmed by PCR using primers mexAB-KO-Chk-F and mexAB-KO-Chk-R (Table S1) and Sanger sequencing. For the $\Delta m e x R$ strain, pEX18Gm:: $\Delta$ mexR was transformed into E. coli SM10( $\lambda$ pir) [53] by TSS transformation, as described previously [54] and selection on LB agar with $20 \mu \mathrm{g} / \mathrm{ml} \mathrm{Gm}$. E. coli SM10( $\lambda$ pir) pEX18Gm:: $\Delta$ mexR was mixed with PAO1, spotted onto an LB plate, and incubated overnight at $30^{\circ} \mathrm{C}$. Matings were collected and plated on VBMM agar with $60 \mu \mathrm{g} / \mathrm{ml}$ Gm to select for $P$. aeruginosa merodiploids and incubated overnight at $37^{\circ} \mathrm{C}$, as described [50]. Merodiploid colonies were streaked onto LB agar with $15 \%$ sucrose and incubated at $25^{\circ} \mathrm{C}$ for $4 \mathrm{~d}$. PAO1 $\Delta$ mexR strains were confirmed by PCR amplification using primers mexR-KO-UP-F and mexR-KO-DN-R (Table S1). To generate the PAO1 $\triangle$ mexEF strain, pDONRPEX18Gm:: $\triangle m e x E F$ was transformed into the donor E. coliS.17.1 $\left(\lambda_{\text {pir }}\right)$ strain. Biparental mating was used to introduce the suicide plasmid into the $P$. aeruginosa PAO1 recipient. Merodiploids resistant to sucrose but sensitive to $\mathrm{Gm}$ were isolated by counterselection on No-Salt LB agar with $15 \% \mathrm{w} / \mathrm{v}$ sucrose after two days of incubation at $30^{\circ} \mathrm{C}$. Colony PCR was performed to identify $\triangle m e x E F$ mutants and PCR products generated 
from the cloned mutant allele were sent for sequencing with oRP_27 and oRP_28 primers. Using this protocol, a $4397 \mathrm{bp}$ fragment from the $4434 \mathrm{bp}$ mexEF sequence was removed resulting in a frameshift mutation in the mexEF-oprN multidrug efflux operon, yielding strain RP05 (PAO1 $\triangle$ mexEF) (Table S1).

\subsection{PAO1 transformation with rhlA reporter plasmid}

To quantify the expression of genes involved in rhamnolipid production, strains PAO1, PAO1-AvEvB8, PAO1 $\triangle$ mexR, PAO1 $\triangle$ mexEF, and PAO1 $\triangle$ mexAB were transformed with $p Y L 122$, a plasmid containing $r h l A-g f p$ promoter fusion in a mini-CTX-lacZ backbone [55]. Following transformation, strains were maintained on LB plates with 100 $\mu \mathrm{g} / \mathrm{mL}$ tetracycline. Clones were then tested using PCR with the primers pYL122-Chk-F and pYL122-Chk-R (Table S1).

\subsection{DNA extraction, purification, and PCR}

Plasmid DNA was prepared using Monarch ${ }^{\circledR}$ Plasmid Miniprep Kit (NEB, cat\# T1010). Genomic DNA was prepared using DNeasy Blood \& Tissue Kit (Qiagen, cat\# 69504). When necessary, cDNA was purified using Monarch ${ }^{\circledR}$ PCR \& DNA Cleanup Kit (NEB, cat\# T1030). PCR was performed using either KAPA HIFI 2X ready mix (KAPA Biosystem, cat\# KK2602).

\subsection{Swarming assay}

Swarming plates were created using $2.5 \% \mathrm{LB}$ and $0.5 \%$ agar. For the assay, all strains were grown overnight in LB broth. For each strain, $2 \mu \mathrm{L}$ of the overnight broth was then placed at the center of a swarming plate and left to incubate at $37^{\circ} \mathrm{C}$ for 24 hours. The maximum swarming diameter was then measured and recorded for each strain.

\subsection{Gradient diffusion antibiotic susceptibility testing}

Aztreonam, ciprofloxacin, and chloramphenicol Etest strips were purchased from bioMérieux (cat\# 501758, 412310, 412308) and antimicrobial susceptibility testing was performed with the following modifications to the manufacturer's instructions. For aztreonam, a sterile swab was soaked into an overnight culture for each strain after growth for $18 \mathrm{~h}$ in LB broth and excess fluid was removed by pressing it against the inside wall of the test tube. For ciprofloxacin and chloramphenicol, colonies were picked directly from LB agar plates and diluted in sterile PBS to OD $600.15\left(\sim 1.5 \times 10^{8} \mathrm{CFU} / \mathrm{ml}\right)$. Mueller Hinton agar plates were fully streaked 4 times with the swabs. After allowing the plates to dry, an Etest gradient strip was placed in the middle of the plates. Plates were incubated at $37^{\circ} \mathrm{C}$ for $16-$ $20 \mathrm{~h}$.

\section{8. rhlA GFP reporter assay}

WT PAO1, PAO1 attB::rhlA-gfp, PAO1-AzEvB8, PAO1-AzEvB8 attB::rhlA-gfp, $\Delta m e x R$, $\triangle m e x R$ attB::rhlA-gfp, $\triangle$ mexEF, $\triangle$ mexEF attB::rhlA-gfp, $\triangle m e x A B$, and $\triangle m e x A B$ attB::rhlA-gfp strains were grown overnight for $16-20 \mathrm{~h}$ in $2 \mathrm{~mL}$ LB. The overnight cultures were then diluted to $\mathrm{OD}_{600} \sim 0.005\left(\sim 5 \times 10^{6} \mathrm{CFU} / \mathrm{mL}\right)$. Two hundred microliters of the working dilutions were added in triplicate to a sterile black (clear bottom) 96-well plate. To prevent evaporation, $50 \mu \mathrm{L}$ of mineral oil was added to each well. The plate was incubated for 24 $\mathrm{h}$ at $37^{\circ} \mathrm{C}$, shaking at $250 \mathrm{rpm}$, and absorbance at $600 \mathrm{~nm}$ and fluorescence with excitation at $488 \mathrm{~nm}$ and emission at $510 \mathrm{~nm}$ were read every hour for $24 \mathrm{~h}$. For each strain, fluorescence was first normalized to the absorbance value. The background of each non-transformed strain was then subtracted from its pYL122-carrying counterpart. Gene expression was calculated as area under the curve using Prism GraphPad. For the efflux pump inhibitor assay, the rhlA reporter assay was performed as described above with the exception that the efflux pump inhibitor PA $\beta N$ (Phe-Arg $\beta$-naphthylamide dihydrochloride, Sigma cat\# P4157-25MG) was added to cultures to reach final concentrations of $0 \mu \mathrm{g} / \mathrm{mL}, 5$ $\mu \mathrm{g} / \mathrm{mL}, 10 \mu \mathrm{g} / \mathrm{mL}$, and $25 \mu \mathrm{g} / \mathrm{mL}$.

\subsection{Murine Lung Infection}

Experiments were approved by the Institutional Animal Care and Use Committee at Cedars-Sinai Medical Center under protocol IACUC008115. Strains were grown to mid- 
exponential phase, washed with sterile PBS, and diluted to $1 \times 10^{8} \mathrm{CFU} / \mathrm{mL}$ in sterile PBS. Female C57BL/6 (Jackson Laboratories) 12-week-old mice (10 mice/group) were anesthetized using isoflurane. A 24-gauge angiocatheter was used to intubate the mice. Acute lung infections were performed by single intratracheal instillations of $5 \times 10^{6} \mathrm{CFUs}$ in $50 \mu \mathrm{L}$ sterile PBS. During inoculation the mice were placed on a surgical board in the supine position. The front paws of each mouse were stretched outwards. A rubber band was placed around the two front incisors of the mouse to help keep their mouths open during the intubation procedure. After inoculation mice were kept in a clean cage under infrared lamp until full recovery. Mice were evaluated thrice per day to assess morbidity, and moribund mice were sacrificed with inhaled $\mathrm{CO}_{2}$. Surviving mice were euthanized $96 \mathrm{~h}$ postinfection. Survival curves were analyzed with log-rank tests as described below.

\subsection{Genome Sequencing and Analysis}

To verify mutations in engineered $P$. aeruginosa strains, DNA was isolated from strains PAO1, PAO1 $\triangle m e x A B$, PAO1-AzEvB8, PAO1-AzEvB8 $\triangle m e x A B$, and PAO1 $\triangle m e x E F$ and subjected to whole genome sequencing. DNA isolation was performed using a DNeasy Blood and Tissue Kit (Qiagen). DNA was submitted to the Microbial Genome Sequencing Center at the University of Pittsburgh where libraries were prepared and sequenced using an Illumina NextSeq platform. Sequencing reads were analyzed using Breseq [56], comparing reads for each strain to the $P$. aeruginosa PAO1 parent reference genome. Figure showing deletion in the $P$. aeruginosa PAO1-AzEvB8 strain was created using CLC Genomics Workbench Software. Genome sequence data will be made available in the NCBI SRA, accession numbers will be provided upon acceptance.

\subsection{Statistics}

Statistical analyses were performed using Prism GraphPad Software v9 and SAS. For swarming and $r h l A$ reporter assays, one-way ANOVA tests were performed followed by Tukey's multiple comparison test. For survival analyses survival times were tested across strata with a log-rank test, with Bonferroni-adjusted p-values where $>2$ strata were compared.

Supplementary Materials: The following are available online at www.mdpi.com/xxx/s1, Table S1: Strains and primers used in this study.

Author Contributions: Conceptualization, M.V. and P.J.; Methodology, M.V. and P.J.; Formal Analysis, M.V., S.P.L., C.B., and P.J.; Investigation, M.V., S.P.L., C.B., R.P., and P.J.; Resources, R.P. and P.J.; Data Curation, M.V., S.P.L., C.B., and P.J.; Writing - Original Draft Preparation, M.V., S.P.L., R.P., and P.J.; Writing - Review \& Editing, M.V., S.P.L., C.B., R.P., and P.J.; Supervision, M.V. and P.J.; Project Administration, M.V. and P.J.; Funding Acquisition, P.J.

Funding: This research was funded by grant numbers JORTH17F5 and JORTH19P0 from the Cystic Fibrosis Foundation and grant numbers K22AI127473 and R01AI14642 from the NIH/National Institute of Allergy and Infectious Diseases, as well as a sub-award from grant number UL1TR001881 from the NIH/National Center for Advancing Translational Science (NCATS) UCLA CTSI.

Institutional Review Board Statement: The study was conducted according to the guidelines of the Declaration of Helsinki and approved by the Institutional Animal Care and Use Committee of Cedars-Sinai Medical Center (protocol code IACUC008115 approved on May 23, 2018).

Informed Consent Statement: Not applicable.

Data Availability Statement: In this section, please provide details regarding where data supporting reported results can be found, including links to publicly archived datasets analyzed or generated during the study. Please refer to suggested Data Availability Statements in section "MDPI Research Data Policies" at https://www.mdpi.com/ethics. You might choose to exclude this statement if the study did not report any data.

Acknowledgments: We would like to thank members of the Jorth Lab, Joe Harrison, and Holly Huse for helpful discussions and feedback on this manuscript. We are grateful to Pradeep K. Singh, Colin Manoil, and Joe J. Harrison for the generous gifts of strains for this study.

Conflicts of Interest: The authors declare no conflict of interest. 


\section{References}

1. Silby, M.W., et al., Pseudomonas genomes: diverse and adaptable. FEMS Microbiol Rev, 2011. 35(4): p. 652-80.

2. Moradali, M.F., S. Ghods, and B.H. Rehm, Pseudomonas aeruginosa Lifestyle: A Paradigm for Adaptation, Survival, and Persistence. Front Cell Infect Microbiol, 2017. 7: p. 39.

3. Morrison, A.J., Jr. and R.P. Wenzel, Epidemiology of infections due to Pseudomonas aeruginosa. Rev Infect Dis, 1984. 6 Suppl 3: p. S627-42.

4. Parkins, M.D., R. Somayaji, and V.J. Waters, Epidemiology, Biology, and Impact of Clonal Pseudomonas aeruginosa Infections in Cystic Fibrosis. Clin Microbiol Rev, 2018. 31(4).

5. Jain, M., et al., Type III secretion phenotypes of Pseudomonas aeruginosa strains change during infection of individuals with cystic fibrosis. J Clin Microbiol, 2004. 42(11): p. 5229-37.

6. Smith, E.E., et al., Genetic adaptation by Pseudomonas aeruginosa to the airways of cystic fibrosis patients. Proc Natl Acad Sci U S A, 2006. 103(22): p. 8487-92.

7. Huse, H.K., et al., Parallel evolution in Pseudomonas aeruginosa over 39,000 generations in vivo. MBio, 2010. 1(4).

8. Jorth, P., et al., Regional Isolation Drives Bacterial Diversification within Cystic Fibrosis Lungs. Cell Host Microbe, 2015. 18(3): p. 30719.

9. Winstanley, C., S. O'Brien, and M.A. Brockhurst, Pseudomonas aeruginosa Evolutionary Adaptation and Diversification in Cystic Fibrosis Chronic Lung Infections. Trends Microbiol, 2016. 24(5): p. 327-337.

10. Darch, S.E., et al., Recombination is a key driver of genomic and phenotypic diversity in a Pseudomonas aeruginosa population during cystic fibrosis infection. Sci Rep, 2015. 5: p. 7649.

11. O'Brien, S., et al., High virulence sub-populations in Pseudomonas aeruginosa long-term cystic fibrosis airway infections. BMC Microbiol, 2017. 17(1): p. 30

12. Faure, E., K. Kwong, and D. Nguyen, Pseudomonas aeruginosa in Chronic Lung Infections: How to Adapt Within the Host? Front Immunol, 2018. 9: p. 2416.

13. Jensen, P.O., et al., Rapid necrotic killing of polymorphonuclear leukocytes is caused by quorum-sensing-controlled production of rhamnolipid by Pseudomonas aeruginosa. Microbiology (Reading), 2007. 153(Pt 5): p. 1329-1338.

14. Zulianello, L., et al., Rhamnolipids are virulence factors that promote early infiltration of primary human airway epithelia by Pseudomonas aeruginosa. Infect Immun, 2006. 74(6): p. 3134-47.

15. Lavoie, E.G., T. Wangdi, and B.I. Kazmierczak, Innate immune responses to Pseudomonas aeruginosa infection. Microbes Infect, 2011. 13(14-15): p. 1133-45.

16. Mogayzel, P.J., Jr., et al., Cystic Fibrosis Foundation pulmonary guideline. pharmacologic approaches to prevention and eradication of initial Pseudomonas aeruginosa infection. Ann Am Thorac Soc, 2014. 11(10): p. 1640-50.

17. Sobel, M.L., et al., Mutations in PA3574 (nalD) lead to increased MexAB-OprM expression and multidrug resistance in laboratory and clinical isolates of Pseudomonas aeruginosa. Antimicrob Agents Chemother, 2005. 49(5): p. 1782-6.

18. Poole, K., et al., Expression of the multidrug resistance operon mexA-mexB-oprM in Pseudomonas aeruginosa: mexR encodes a regulator of operon expression. Antimicrob Agents Chemother, 1996. 40(9): p. 2021-8.

19. Saito, K., H. Yoneyama, and T. Nakae, nalB-type mutations causing the overexpression of the MexAB-OprM efflux pump are located in the mexR gene of the Pseudomonas aeruginosa chromosome. FEMS Microbiol Lett, 1999. 179(1): p. 67-72.

20. Jorth, P., et al., Evolved Aztreonam Resistance Is Multifactorial and Can Produce Hypervirulence in Pseudomonas aeruginosa. MBio, 2017. 8(5).

21. Suresh, M., et al., Mutational analyses of regulatory genes, mexR, nalC, nalD and mexZ of mexAB-oprM and mexXY operons, in efflux pump hyperexpressing multidrug-resistant clinical isolates of Pseudomonas aeruginosa. World J Microbiol Biotechnol, 2018. 34(6): $\mathrm{p}$. 83.

22. Horna, G., et al., Interplay between MexAB-OprM and MexEF-OprN in clinical isolates of Pseudomonas aeruginosa. Sci Rep, 2018. 8(1): p. 16463.

23. Llanes, C., et al., Clinical strains of Pseudomonas aeruginosa overproducing MexAB-OprM and MexXY efflux pumps simultaneously. Antimicrob Agents Chemother, 2004. 48(5): p. 1797-802.

24. McLean, K., et al., Genomic Analysis Identifies Novel Pseudomonas aeruginosa Resistance Genes under Selection during Inhaled Aztreonam Therapy In Vivo. Antimicrob Agents Chemother, 2019. 63(9).

25. Oshri, R.D., et al., Selection for increased quorum-sensing cooperation in Pseudomonas aeruginosa through the shut-down of a drug resistance pump. ISME J, 2018. 12(10): p. 2458-2469.

26. Kohler, T., et al., Overexpression of the MexEF-OprN multidrug efflux system affects cell-to-cell signaling in Pseudomonas aeruginosa. J Bacteriol, 2001. 183(18): p. 5213-22.

27. Lomovskaya, O., et al., Identification and characterization of inhibitors of multidrug resistance efflux pumps in Pseudomonas aeruginosa: novel agents for combination therapy. Antimicrob Agents Chemother, 2001. 45(1): p. 105-16.

28. Cosson, P., et al., Pseudomonas aeruginosa virulence analyzed in a Dictyostelium discoideum host system. J Bacteriol, 2002. 184(11): $\mathrm{p}$. 3027-33.

29. Hirakata, Y., et al., Efflux pump inhibitors reduce the invasiveness of Pseudomonas aeruginosa. Int J Antimicrob Agents, 2009. 34(4): p. 343-6.

30. Rampioni, G., et al., Effect of efflux pump inhibition on Pseudomonas aeruginosa transcriptome and virulence. Sci Rep, 2017. 7(1): p. 11392. 
31. Miyata, S., et al., Use of the Galleria mellonella caterpillar as a model host to study the role of the type III secretion system in Pseudomonas aeruginosa pathogenesis. Infect Immun, 2003. 71(5): p. 2404-13.

32. Kropinski, A.M. and J.S. Chadwick, The pathogenicity of rough strains of Pseudomonas aeruginosa for Galleria mellonella. Can J Microbiol, 1975. 21(12): p. 2084-8.

33. Marvig, R.L., et al., Convergent evolution and adaptation of Pseudomonas aeruginosa within patients with cystic fibrosis. Nat Genet, 2015. 47(1): p. 57-64.

34. Fukuda, H., et al., New norfloxacin resistance gene in Pseudomonas aeruginosa PAO. Antimicrob Agents Chemother, 1990. 34(9): p. 1757-61.

35. Kohler, T., et al., Characterization of MexE-MexF-OprN, a positively regulated multidrug efflux system of Pseudomonas aeruginosa. Mol Microbiol, 1997. 23(2): p. 345-54.

36. Quale, J., et al., Interplay of efflux system, ampC, and oprD expression in carbapenem resistance of Pseudomonas aeruginosa clinical isolates. Antimicrob Agents Chemother, 2006. 50(5): p. 1633-41.

37. Tomas, M., et al., Efflux pumps, OprD porin, AmpC beta-lactamase, and multiresistance in Pseudomonas aeruginosa isolates from cystic fibrosis patients. Antimicrob Agents Chemother, 2010. 54(5): p. 2219-24.

38. Keating, C.L., et al., Pseudomonas aeruginosa Susceptibility Patterns and Associated Clinical Outcomes in People with Cystic Fibrosis following Approval of Aztreonam Lysine for Inhalation. Antimicrob Agents Chemother, 2021. 65(3).

39. Yoshida, K., et al., MexAB-OprM specific efflux pump inhibitors in Pseudomonas aeruginosa. Part 7: highly soluble and in vivo active quaternary ammonium analogue D13-9001, a potential preclinical candidate. Bioorg Med Chem, 2007. 15(22): p. 7087-97.

40. Roux, D., et al., Fitness cost of antibiotic susceptibility during bacterial infection. Sci Transl Med, 2015. 7(297): p. $297 \mathrm{ra114.}$

41. Cowley, E.S., et al., Pediatric Cystic Fibrosis Sputum Can Be Chemically Dynamic, Anoxic, and Extremely Reduced Due to Hydrogen Sulfide Formation. MBio, 2015. 6(4): p. e00767.

42. Palmer, K.L., S.A. Brown, and M. Whiteley, Membrane-bound nitrate reductase is required for anaerobic growth in cystic fibrosis sputum. J Bacteriol, 2007. 189(12): p. 4449-55.

43. Palmer, K.L., L.M. Aye, and M. Whiteley, Nutritional cues control Pseudomonas aeruginosa multicellular behavior in cystic fibrosis sputum. J Bacteriol, 2007. 189(22): p. 8079-87.

44. Turner, K.H., et al., Essential genome of Pseudomonas aeruginosa in cystic fibrosis sputum. Proc Natl Acad Sci U S A, 2015. 112(13): p. 4110-5.

45. Rogers, C.S., et al., Disruption of the CFTR gene produces a model of cystic fibrosis in newborn pigs. Science, 2008. 321(5897): p. 183741.

46. Starke, J.R., et al., A mouse model of chronic pulmonary infection with Pseudomonas aeruginosa and Pseudomonas cepacia. Pediatr Res, 1987. 22(6): p. 698-702.

47. van Heeckeren, A.M. and M.D. Schluchter, Murine models of chronic Pseudomonas aeruginosa lung infection. Lab Anim, 2002. 36(3): p. 291-312.

48. van Heeckeren, A.M., et al., Role of Cftr genotype in the response to chronic Pseudomonas aeruginosa lung infection in mice. Am J Physiol Lung Cell Mol Physiol, 2004. 287(5): p. L944-52.

49. Held, K., et al., Sequence-verified two-allele transposon mutant library for Pseudomonas aeruginosa PAO1. J Bacteriol, 2012. 194(23): p. 6387-9.

50. Hmelo, L.R., et al., Precision-engineering the Pseudomonas aeruginosa genome with two-step allelic exchange. Nat Protoc, 2015. 10(11): p. 1820-41.

51. Hoang, T.T., et al., A broad-host-range Flp-FRT recombination system for site-specific excision of chromosomally-located DNA sequences: application for isolation of unmarked Pseudomonas aeruginosa mutants. Gene, 1998. 212(1): p. 77-86.

52. Taylor, R.G., D.C. Walker, and R.R. McInnes, E. coli host strains significantly affect the quality of small scale plasmid DNA preparations used for sequencing. Nucleic Acids Res, 1993. 21(7): p. 1677-8.

53. Simon, R., Priefer, U. \& Pühler, A. , A Broad Host Range Mobilization System for In Vivo Genetic Engineering: Transposon Mutagenesis in Gram Negative Bacteria. Nat Biotechnol, 1983. 1: p. 784-791.

54. Chung, C.T., S.L. Niemela, and R.H. Miller, One-step preparation of competent Escherichia coli: transformation and storage of bacterial cells in the same solution. Proc Natl Acad Sci U S A, 1989. 86(7): p. 2172-5.

55. Lequette, Y. and E.P. Greenberg, Timing and localization of rhamnolipid synthesis gene expression in Pseudomonas aeruginosa biofilms. J Bacteriol, 2005. 187(1): p. 37-44.

56. Deatherage, D.E. and J.E. Barrick, Identification of mutations in laboratory-evolved microbes from next-generation sequencing data using breseq. Methods Mol Biol, 2014. 1151: p. 165-88. 